\title{
Impact of Deploying a Genetic Approach to Stem Cells Opens-Up New Facets in the "Blank Slates" of Our Body
}

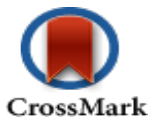

\author{
Jyoti Bhojwani*
}

School of Life Sciences, DAVV/Indore University, Indore, M.P, India

Corresponding Author: Dr. Jyoti Bhojwani, School of Life Sciences, DAVV/Indore University, Indore, M.P, India. E-mail: jbhojwani2005@gmail.com

\section{Received Date: October 8, 2015 Accepted Date: November 19, 2015 Published Date: November 27,2015}

Citation: Bhojwani, J. Impact of Deploying a Genetic Approach to Stem Cells Opens-Up New Facets in the "Blank Slates" of Our Body. (2015) J Stem Cell Regen Bio 1(1): 16- 18.

\section{Introduction}

Since the dawn of the Post-Genomic era (25 years back), applying a genetic approach to solving various intricate problems/ issues in research has taken-off even more swiftly than ever before! This commentary briefs the major impact of such an approach on the some of the advances in stem cell biology and innovations in various clinical applications, primarily focusing on "Cancer Stem Cells" (CSCs), which have been the major targets for cancer therapy in recent times. The touchdown is basically toward understanding very fundamental aspects/applications of stem cells ${ }^{[1-3]}$, including both embryonic and adult stem cells from the histological discovery to the present day scenario. I have presented and exemplified "Cancer Stem Cells" here, to contrast normal developmental and maintenance types of stem cells, in the understanding of some prominent pathways and molecular interactions through genetic approaches, which may consequently lead to unveiling the underlying mechanisms of malignant transformation and tumorigenesis. This will presumably aid in innovating powerful ways to identify therapeutic targets for cancer patients in near future.

Spatio-temporal cues defined for certain critical components in a particular developmental pathway (involved in causing/ progression of certain disease) provide a firm basis for detecting the order, hierarchy and "switching-off or on" of genes that regulate it. The various time-points, at which genes are switched on/off, clearly determines the fate of what a cell does in terms of being functional or non-functional, due to disruption of that specific pathway. Recent research-work in this area ${ }^{[4]}$ provides strong evidence, toward identifying such components (associated with Wnt-signaling involved in Colorectal Cancer-CRC disease). These crucial elements indeed determine the genetic transformation of a "blank-slate" ("cells of origin" and/or putative "cancer stem cells") or "primitive-state" epithelial cells to an intermediate adenoma/polyp (dyspastic), and later to a proliferative (hyperplastic) or cancerous (neoplastic) state. Citing this report of mine and some more, the idea is to re-iterate the power of genetics, in solving and filling the missing links of any developmental pathway (in question) involved in progression of a disease (in this case, CRC). A critical temporal requirement of certain molecules [Caesin-Kinase I (CKI) and Human-Discs-large (hDlg)] was finally established in this particular study, and, these proteins were identified as "early" and "late" acting molecules respectively, in a very crucial developmental event, that basically transforms "polyps" to full-fledged "carcinomas" (epithelial cancers) in COLORECTAL tumors. The detection of these genetic and developmental parameters (as the "cause"), served as a focal-point and a prominent diagnostic feature, for consequential detection of "effects", i.e., gain/loss of other related components involved during progression of CRC disease. Coincidentally, the chromosomes on which these genes reside, have also been found to be dense and rich in Single Nucleotide Polymorphisms; SNPs (hot-spots), the details of which were published in a separate report ${ }^{[5]}$. This study has also been considered to be "one of the kind" by the scientific community, since it harnessed the potential of Genetics, Developmental Biology and Bio-Informatics tools (in combination, for the first time), to solve a long-standing puzzle in pin-pointing some crucial genetic factors, that were critically involved in the progression of CRC disease. This report has created enough impact, in terms of authentically suggesting, that it is only when we deploy a combinatorial approach towards certain complicated biological problems, can we successfully unveil the underlying mechanisms in greater details. 
At the heart of every tumor, researchers now believe, lie a handful of aberrant stem cells (referred to as: "Cancer Stem Cells" or CSCs), that maintain the malignant tissue, and actually define the "cells of origin" from where the cancers emerge or stem from. This logic, if correct, could explain why tumors often regenerate even after being almost destroyed by anti-cancer drugs. It also hints on the deployment of a different strategy for developing anti-cancer drugs, suggesting that, these should be selected for lethality to CSCs and not, as it is at present, for their ability to kill just any cells in the body and shrink tumors. Cancer cells (hyperplastic: benign; neoplastic: malignant) are known for their unlimited cell division capacity, reminiscent of self-renewing proliferation, characteristic of stem cells. Recent data also indicates that the transformation process exploits a unique physiological dependence of intestinal progenitor/stem cell on the Wnt (counterpart of wingless in Drosophila) cascade, thus pointing to the fact, that, Wnt signaling pathway may thus be a common element in regulating stem cell renewal and/or maintenance in a variety of systems ${ }^{[6]}$.

Although, since both govern opposite functions - "Stem cells" of life and "Cancer cells" of death, there are striking differences between the two. First, the self-renewing machinery in stem cells responds to a feedback mechanism (Figure1), which senses the number of mature cells and regulates rate of division, whereas in cancer cells, this mechanism stands totally disrupted. Second, unlike stem cells, which can differentiate into three germ layers (teratomas), high-grade tumor cells lack the ability to properly differentiate into mature cells. Lastly, tumor cells are prone to accumulate replication errors, while somatic stem cells develop mechanisms that guard against this. Their differences apart, they both posses and exhibit high and continuous proliferative/regenerative potential, a strikingly obvious common feature, due to which, it is easier for tumors to "hijack" stem cells that have essential machinery already in place. It has long been speculated that there is a unique sub-population in tumor cells with stem cell-like properties (now known as "Cancer Stem Cells" or "Bad seeds"; abbreviated as CSCs) and that. tumorigenesis occurs at stem cell level[ ${ }^{[7]}$. Since no definitive markers or efficient labeling tools are available, this population of cells still remains elusive in both cancer and stem cell biology. Therefore, it would be critical to understand molecular differences between stem cells and cancer cells, which might be helpful in providing novel insights into the mechanism of tumorigenesis as well as potential therapeutic targets, in foreseeable future. We have come a long way in the stem cell advances over time (briefed in Table1). Very recent breakthroughs include: (a) The tuning and genetic re-programming of stem cells ( $i P S$ cells) by a handful of genetic factors ${ }^{[8-10]}$ and; (b) The transformation of cancerous cells to normal cells by reversing the genetic changes involved and also restricting the awry cancerous cells by using microRNAs (https://www.rt.com/news/313297-cancer-medicine-science-biology/), deemed to be as a major leap forward for Cancer researchers (http://yournewswire.com/breakthroughscientists-find-way-to-change-cancer-cells-into-healthy-cells/). However, it still remains to be seen, how we can intelligently utilize these efficient innovative tools together, to attack the "Bad seeds" and actually "find a cure", rather than "just talking about" ways to cure the myriad diseases, like Cancer. Of course, other important functional aspects of embryonic and adult stem cells include; differentiation, regeneration, repair, immune modula- tion and paracrine effects. These effects are indeed very powerful and can be aptly utilized in a variety of clinical applications, including; transplantation, autoimmune disease, neuro-degeneration, diabetes, aging problems, etc., pending promising research/clinical trials. But once this is eventually achieved, we may further be able to harness the potential of stem cells for effective diagnosis, prognosis and identifying therapeutic targets not only for cancer patients, but for patients suffering from other diseases/accidental damage/impairments, as well.

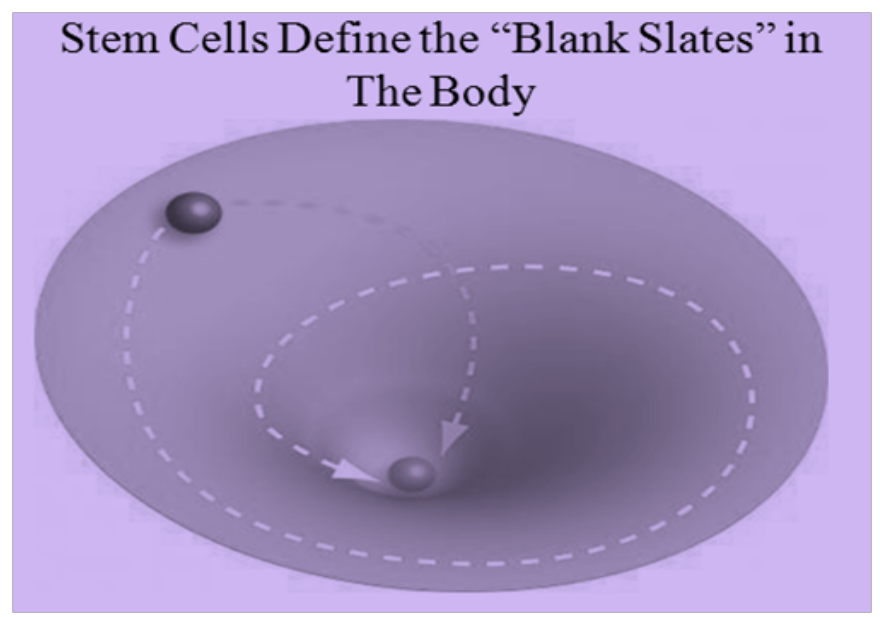

Figure 1: Stem Cells define the major "Blank Slates" in the body, which can be tuned to Genetic conditioning for curing various diseases/ impairments!

Table 1: Stem Cell Timeline/ Milestones

\begin{tabular}{|l|l|}
\hline Year & Milestone-Achievements \\
\hline 1956 & First successful bone marrow transplant \\
\hline 1981 & Embryonic stem cells (ESCs) isolated from mouse blastocysts \\
\hline 1988 & $\begin{array}{l}\text { Hematopoietic stem cells from adult mice were purified and } \\
\text { characterized }\end{array}$ \\
\hline 1992 & Stem cells were identified in adult human brain \\
\hline 1998 & The first Human embryonic stem cells (HESCs) were identified \\
\hline 2007 & Genetic re-programming of Stem Cells (iPS cells) \\
\hline 2013 & Cloning of ESCs using DNA from skin cells \\
\hline 2015 & $\begin{array}{l}\text { Prospects of genetically tuning the embryonic and adult stem } \\
\text { cells to combat myriad diseases }\end{array}$ \\
\hline
\end{tabular}




\section{References}

1. Bhojwani, J. Tailor-Made Stem cells. (2006) Current Science 90(10): 1311-1314.

2. Bhojwani, J. Ethics in Scientific Research. (2006) Current Science 90 (10): 1307

3. Bhojwani, J. Stem cells derived without destruction of embryos: A breakthrough study or a study breaking the backbone of science. (2006) Current Science 91(12): 1597.

4. Bhojwani, J. Comparative expression profiling reveals a critical temporal requirement of $\mathrm{CKI}$ and $\mathrm{hDlg}$ in developing colorectal carcinomas. (2015) [in press]

5. Patidar, P., Bhojwani, J. Identification and Pattern Analysis of SNPs involved in Colorectal Cancer. (2013) Journal of Stem Cell Research and Therapy 3(3): 144-150.

6. Taipale., Beachy. The Hedgehog and Wnt signaling pathways in cancer. (2001) Nature 411: 349-354.

7. Reya, T., Morrison, S.J., Clarke, M.E., et al. Stem cells, Cancer, Cancer stem cells. (2001) Nature 414: 105-111

8. Takahashi, K., Tanabe, K., Ohnuki, M., et al. Induction of pluripotent stem cells from adult human fibroblasts by defined factors. (2007) Cell 131(5): 861-872.

9. Yu, J., Vodyanik, M.A., Smuga-Otto, K., et al. Induced pluripotent stem cell lines derived from human somatic cells. (2007) Science 318(5858): 1917-1920.

10. Bhojwani, J. Genetically Reprogrammed Skin Cells bypassing the need for Embryonic Stem Cells - A Ray of Hope for Ethical Science!! (2007) Current Science 94(4): 435.

Ommega Online Publisher Journal of Stem Cell \& Regenerative Biology Short Title : J Stem Cell Regen Bio 